\title{
Identity Method for the Determination of the Moments of Multiplicity Distributions
}

\author{
A. Rustamov ${ }^{1}$ and M. I. Gorenstein ${ }^{2,3}$ \\ ${ }^{1}$ Institut für Kernphysik, Johann Wolfgang Goethe Universität Frankfurt, Germany \\ ${ }^{2}$ Bogolyubov Institute for Theoretical Physics, Kiev, Ukraine \\ ${ }^{3}$ Frankfurt Institute for Advanced Studies, Frankfurt, Germany
}

\begin{abstract}
Recently the identity method was proposed to calculate second moments of the multiplicity distributions from event-by-event measurements in the presence of the effects of incomplete particle identification. In this paper the method is extended for higher moments. The moments of smeared multiplicity distributions are calculated using single-particle identity variables. The problem of finding the moments of the multiplicity distributions is reduced to solving of a system of linear equations.
\end{abstract}




\section{INTRODUCTION}

The study of event-by-event fluctuations of chemical (particle-type) composition in high energy nucleus-nucleus collisions is a helpful tool to pin-down the properties of strongly interacting matter (see, e.g., review [1] and references therein). By measuring the fluctuations one may observe anomalies associated with transition between hadronic and partonic phases. Furthermore, the QCD critical point may be signalled by a characteristic pattern in the fluctuations [2 4]. Data on event-by-event chemical fluctuations from the CERN SPS [5-7] and BNL RHIC [8] were already published, and more systematic measurements are in progress.

A serious experimental problem of event-by-event determination of the multiplicity of different hadron species is an incomplete particle identification caused by finite detector resolution. However, one can usually determine distributions $\rho_{j}(x)$ of an identification observable $x$ and obtain the average multiplicities $\left\langle N_{j}\right\rangle$ for different hadron species $j$.

As an artefact of particle mis-identification measured fluctuations may be systematically reduced or enlarged. To overcome this problem a new experimental technique, called the identity method, was proposed in Ref. 9] for the analysis of events with two measured particle species. In Ref. [10] this method was further developed to calculate the second moments of the multiplicity distributions of more than two particle species. In the present study we prove that the identity method can be generalized to determine third and higher moments of the multiplicity distributions in events consisting of an arbitrary number of different particle species. It was already emphasized that measurements of the third and higher moments for the event-by-event fluctuations is important for the search of critical point signals in nucleus-nucleus collisions [11, 12].

The paper is organized as follows. The used notations and definitions are introduced in Section III. The identity method for the second moments of the multiplicity distribution is reviewed in Section III. In Section IV the method is generalized to third and higher moments of the multiplicity distribution. We consider in Section $\mathrm{V}$ the case in which the normalization of mass distributions are unknown, and finally in section VI we test the developed formalism in simulation. Section VII summarizes the paper. 


\section{NOTATIONS AND DEFINITIONS}

We assume that particle identification (PID) is achieved by measuring a quantity $x^{1}$. Since any measurement is of finite resolution, we deal with continuous distributions of $x$ denoted by $\rho_{j}(x)(j=1, \ldots, k \geq 2$, with $k$ being the number of different particle types) and normalized as

$$
\int d x \rho_{j}(x)=\left\langle N_{j}\right\rangle
$$

where $\left\langle N_{j}\right\rangle$ is the mean multiplicity of the particle type $j$. Note that the functions $\rho_{j}(x)$ are found for the different particle species using the inclusive distribution of $x$ for all particles in all events. The identity variables $w_{j}(x)$ are defined as:

$$
w_{j}(x) \equiv \frac{\rho_{j}(x)}{\rho(x)}
$$

where

$$
\rho(x) \equiv \sum_{j=1}^{k} \rho_{j}(x)
$$

The complete identification (CI) of particles corresponds to distributions $\rho_{j}(x)$, which do not overlap. This case leads to $w_{j}=0$ for all particle species $i \neq j$ and $w_{j}=1$ for the $j^{\text {th }}$ species. When the distributions $\rho_{j}(x)$ overlap, $w_{j}(x)$ can take any real value between 0 and 1 .

We further introduce the event quantities, $W_{j}$, for each particle type $j$ :

$$
W_{j} \equiv \sum_{i=1}^{N(n)} w_{j}\left(x_{i}\right)
$$

with $j=1, \cdots, k$, and define the event averages as

$$
\left\langle W_{p} \cdot \ldots \cdot W_{q}\right\rangle=\frac{1}{N_{\mathrm{ev}}} \sum_{n=1}^{N_{\mathrm{ev}}} W_{p} \cdot \ldots \cdot W_{q},
$$

where $N_{\mathrm{ev}}$ is the number of events and $N(n)$ is the total multiplicity in the $n^{\text {th }}$ event. Each experimental event is characterized by a set of measured values of PID variable $x\left\{x_{1}, x_{2}, \ldots, x_{N}\right\}$, for which one can calculate the identity variables: $\left\{w_{j}\left(x_{1}\right), w_{j}\left(x_{2}\right), \ldots, w_{j}\left(x_{N}\right)\right\}$, with $j=$

\footnotetext{
${ }^{1}$ Here by $x$ we refer to any experimentally measured particle property related to its identity, e.g. specific energy loss in a detector gas.
} 
$1, \ldots, k$. Consequently, the quantities $W_{j}, W_{j}^{2}, W_{p} W_{q}$ etc. are uniquely defined for each event, and their average values (see Eq. (5) ) can be found experimentally by straightforward averaging over all events. In the case of CI, one finds $W_{j}=N_{j}$, thus, Eq. (5) yields

$$
\left\langle W_{p} \cdot \ldots \cdot W_{q}\right\rangle=\left\langle N_{p} \cdot \ldots \cdot N_{q}\right\rangle
$$

The $W$-quantities are the event variables as they are calculated for each event. By averaging different combinations of these quantities over events, the moments of the $W$-distributions $\left\langle W_{p} \ldots W_{q}\right\rangle$ can be easily reconstructed directly from experimental data. However, the goal is to obtain the moments $\left\langle N_{p} \ldots N_{q}\right\rangle$ of the multiplicity distribution, which are unknown in the case of incomplete particle identification. The main idea here is to find the relation between the moments of the $W$-quantities calculated from the measurements and the unknown moments of the multiplicity distribution.

\section{SECOND MOMENTS}

The relation between second moments of multiplicity distributions and corresponding second moments of $W$ s has been obtained in Ref. [10]. The quantities $\left\langle W_{j}^{2}\right\rangle$ and $\left\langle W_{p} W_{q}\right\rangle$ were found as specific linear combinations of all first and second moments $\left\langle N_{j}\right\rangle$ and $\left\langle N_{j}^{2}\right\rangle$, as well as the correlation terms $\left\langle N_{p} N_{q}\right\rangle$. As the first moments are given by Eq. (1) the mathematical problem of finding of all the second moments $\left\langle N_{j}^{2}\right\rangle$ and $\left\langle N_{p} N_{q}\right\rangle$ is then reduced to solving a system of linear equations.

\section{THIRD AND HIGHER MOMENTS}

In this section we derive formulae for all pure and mixed third moments of the unknown multiplicity distributions. Following the procedure developed for the second moments (see the previous section) we introduce the multiplicity $\left(\mathcal{P}\left(N_{1}, \ldots, N_{k}\right)\right)$ and the PID probability density $\left(P_{i}(x) \equiv \rho_{i}(x) /\left\langle N_{i}\right\rangle\right)$ functions. Using these functions we get:

$$
\left\langle W_{p}^{3}\right\rangle=\sum_{N_{1}=0}^{N_{1}=\infty} \sum_{N_{2}=0}^{N_{2}=\infty} \ldots \sum_{N_{k}=0}^{N_{k}=\infty} \mathcal{P}\left(N_{1}, N_{2}, \ldots, N_{k}\right) \int d x_{1}^{1} P_{1}\left(x_{1}^{1}\right) \ldots \int d x_{N_{1}}^{1} P_{1}\left(x_{N_{1}}^{1}\right)
$$




$$
\begin{aligned}
& \times \int d x_{1}^{2} P_{2}\left(x_{1}^{2}\right) \ldots \int d x_{N_{2}}^{2} P_{2}\left(x_{N_{2}}^{2}\right) \times \ldots \times \int d x_{1}^{k} P_{k}\left(x_{1}^{k}\right) \ldots \times \int d x_{N_{k}}^{k} P_{k}\left(x_{N_{k}}^{k}\right) \\
& \times\left[w_{p}\left(x_{1}^{1}\right)+\ldots+w_{p}\left(x_{N_{1}}^{1}\right)+w_{p}\left(x_{1}^{2}\right)+\ldots+w_{p}\left(x_{N_{2}}^{2}\right)+w_{p}\left(x_{1}^{k}\right)+\ldots+w_{p}\left(x_{N_{k}}^{k}\right)\right]^{3} \\
& =\sum_{i=1}^{k}\left\langle N_{i}^{3}\right\rangle{\overline{w_{p, i}}}^{3}+3 \sum_{1 \leq i<l \leq k}\left\langle N_{i}^{2} N_{l}\right\rangle\left(\overline{w_{p, i}} \cdot \overline{w_{p, l}}\right)+3 \sum_{1 \leq i<l \leq k}\left\langle N_{i} N_{l}^{2}\right\rangle\left(\overline{w_{p, l}} \cdot \overline{w_{p, i}}\right) \\
& +6 \sum_{1 \leq i<l<m \leq k}\left\langle N_{i} N_{l} N_{m}\right\rangle W_{i l m}^{p p p}+3 \sum_{i=1}^{k}\left\langle N_{i}^{2}\right\rangle\left(\overline{w_{p, i}^{2}} \cdot \overline{w_{p, i}}-{\overline{w_{p, i}}}^{3}\right) \\
& +3 \sum_{1 \leq i<l \leq k}\left\langle N_{i} N_{l}\right\rangle\left(\overline{w_{p, i}^{2}} \cdot \overline{w_{p, l}}+\overline{w_{p, l}^{2}} \cdot \overline{w_{p, i}}-\overline{w_{p, i}} \cdot \overline{w_{p, l}}\right. \\
& \left.-{\overline{w_{p, l}}}^{2} \cdot \overline{w_{p, i}}\right)+\sum_{i=1}^{k}\left\langle N_{i}\right\rangle\left(2 \overline{w_{p, i}} 3+\overline{w_{p, i}^{3}}-3 \overline{w_{p, i}^{2}} \cdot \overline{w_{p, i}}\right)
\end{aligned}
$$

$$
\begin{aligned}
& \left\langle W_{p} W_{q} W_{r}\right\rangle=\sum_{N_{1}=0}^{N_{1}=\infty} \sum_{N_{2}=0}^{N_{2}=\infty} \ldots \sum_{N_{k}=0}^{N_{k}=\infty} \mathcal{P}\left(N_{1}, N_{2}, \ldots, N_{k}\right) \int d x_{1}^{1} P_{1}\left(x_{1}^{1}\right) \ldots \int d x_{N_{1}}^{1} P_{1}\left(x_{N_{1}}^{1}\right) \\
& \times \int d x_{1}^{2} P_{2}\left(x_{1}^{2}\right) \ldots \int d x_{N_{2}}^{2} P_{2}\left(x_{N_{2}}^{2}\right) \times \ldots \times \int d x_{1}^{k} P_{k}\left(x_{1}^{k}\right) \ldots \int d x_{N_{k}}^{k} P_{k}\left(x_{N_{k}}^{k}\right) \\
& \times\left[w_{p}\left(x_{1}^{1}\right)+\ldots+w_{p}\left(x_{N_{1}}^{1}\right)+w_{p}\left(x_{1}^{2}\right)+\ldots+w_{p}\left(x_{N_{2}}^{2}\right)+w_{p}\left(x_{1}^{k}\right)+\ldots+w_{p}\left(x_{N_{k}}^{k}\right)\right] \\
& \times\left[w_{q}\left(x_{1}^{1}\right)+\ldots+w_{q}\left(x_{N_{1}}^{1}\right)+w_{q}\left(x_{1}^{2}\right)+\ldots+w_{q}\left(x_{N_{2}}^{2}\right)+w_{q}\left(x_{1}^{k}\right)+\ldots+w_{q}\left(x_{N_{k}}^{k}\right)\right] \\
& \times\left[w_{r}\left(x_{1}^{1}\right)+\ldots+w_{r}\left(x_{N_{1}}^{1}\right)+w_{r}\left(x_{1}^{2}\right)+\ldots+w_{r}\left(x_{N_{2}}^{2}\right)+w_{r}\left(x_{1}^{k}\right)+\ldots+w_{r}\left(x_{N_{k}}^{k}\right)\right] \\
& =\sum_{i=1}^{k}\left\langle N_{i}^{3}\right\rangle W_{i i i}^{p q r}+\sum_{1 \leq i<l \leq k}\left\langle N_{i}^{2} N_{l}\right\rangle\left(W_{i i l}^{p q r}+W_{i l i}^{p q r}+W_{l i i}^{p q r}\right) \\
& +\sum_{1 \leq i<l \leq k}\left\langle N_{i} N_{l}^{2}\right\rangle\left(W_{l l i}^{p q r}+W_{l i l}^{p q r}+W_{i l l}^{p q r}\right) \\
& +\sum_{1 \leq i<l<m \leq k}\left\langle N_{i} N_{l} N_{m}\right\rangle\left(W_{i l m}^{p q r}+W_{l m i}^{p q r}+W_{m i l}^{p q r}+W_{i m l}^{p q r}+W_{l i m}^{p q r}+W_{m l i}^{p q r}\right) \\
& +\sum_{i=1}^{k}\left\langle N_{i}^{2}\right\rangle\left(\overline{w_{p q, i}} \cdot \overline{w_{r, i}}+\overline{w_{p r, i}} \cdot \overline{w_{q, i}}+\overline{w_{q r, i}} \cdot \overline{w_{p, i}}-3 \overline{w_{p, i}} \cdot \overline{w_{q, i}} \cdot \overline{w_{r, i}}\right) \\
& +\sum_{1 \leq i<l \leq k}\left\langle N_{i} N_{l}\right\rangle\left(W_{i l}^{(p q) r}+W_{l i}^{(p q) r}+W_{i l}^{(p r) q}+W_{l i}^{(p r) q}+W_{i l}^{(q r) p}+W_{l i}^{(q r) p}\right) \\
& +\sum_{i=1}^{k}\left\langle N_{i}\right\rangle\left(\overline{w_{p q r, i}}+2 W_{i i i}^{p q r}-\overline{w_{p q, i}} \cdot \overline{w_{r, i}}-\overline{w_{p r, i}} \cdot \overline{w_{q, i}}-\overline{w_{q r, i}} \cdot \overline{w_{p, i}}\right)
\end{aligned}
$$




$$
\begin{aligned}
& \left\langle W_{p}^{2} W_{q}\right\rangle=\sum_{N_{1}=0}^{N_{1}=\infty} \sum_{N_{2}=0}^{N_{2}=\infty} \ldots \sum_{N_{k}=0}^{N_{k}=\infty} \mathcal{P}\left(N_{1}, N_{2}, \ldots, N_{k}\right) \int d x_{1}^{1} P_{1}\left(x_{1}^{1}\right) \ldots \int d x_{N_{1}}^{1} P_{1}\left(x_{N_{1}}^{1}\right) \\
& \times \int d x_{1}^{2} P_{2}\left(x_{1}^{2}\right) \ldots \int d x_{N_{2}}^{2} P_{2}\left(x_{N_{2}}^{2}\right) \times \ldots \times \int d x_{1}^{k} P_{k}\left(x_{1}^{k}\right) \ldots \\
& \times \int d x_{N_{k}}^{k} P_{k}\left(x_{N_{k}}^{k}\right)\left[w_{p}\left(x_{1}^{1}\right)+\ldots+w_{p}\left(x_{N_{1}}^{1}\right)+w_{p}\left(x_{1}^{2}\right)+\ldots+w_{p}\left(x_{N_{2}}^{2}\right)+w_{p}\left(x_{1}^{k}\right)+\ldots+w_{p}\left(x_{N_{k}}^{k}\right)\right]^{2} \\
& \times\left[w_{q}\left(x_{1}^{1}\right)+\ldots+w_{q}\left(x_{N_{1}}^{1}\right)+w_{q}\left(x_{1}^{2}\right)+\ldots+w_{q}\left(x_{N_{2}}^{2}\right)+w_{q}\left(x_{1}^{k}\right)+\ldots+w_{q}\left(x_{N_{k}}^{k}\right)\right] \\
& =\sum_{i=1}^{k}\left\langle N_{i}^{3}\right\rangle{\overline{w_{p, i}}}^{2} \cdot \overline{w_{q, i}}+\sum_{1 \leq i<l \leq k}\left\langle N_{i}^{2} N_{l}\right\rangle\left(\overline{w_{p, i}} \cdot \overline{w_{q, l}}+2 W_{i l i}^{p p q}\right)+\sum_{1 \leq i<l \leq k}\left\langle N_{i} N_{l}^{2}\right\rangle\left(\overline{w_{p, l}} \cdot \overline{w_{q, i}}+2 W_{i l l}^{p p q}\right) \\
& +2 \sum_{1 \leq i<l<m \leq k}\left\langle N_{i} N_{l} N_{m}\right\rangle\left(W_{i l m}^{p p q}+W_{i m l}^{p p q}+W_{l m i}^{p p q}\right)+\sum_{i=1}^{k}\left\langle N_{i}^{2}\right\rangle\left(\overline{w_{p, i}^{2}} \cdot \overline{w_{q, i}}-3{\overline{w_{p, i}}}^{2} \cdot \overline{w_{q, i}}+2 \overline{w_{q p, i}} \cdot \overline{w_{p, i}}\right) \\
& +\sum_{1 \leq i<l \leq k}\left\langle N_{i} N_{l}\right\rangle\left(2 W_{i l}^{(p q) p}+2 W_{l i}^{(p q) p}+\overline{w_{p, i}^{2}} \cdot \overline{w_{q, l}}+\overline{w_{p, l}^{2}} \cdot \overline{w_{q, i}}-{\overline{w_{p, i}}}^{2} \cdot \overline{w_{q, l}}-{\overline{w_{p, l}}}^{2} \cdot \overline{w_{q, i}}\right) \\
& +\sum_{i=1}^{k}\left\langle N_{i}\right\rangle\left(2{\overline{w_{p, i}}}^{2} \cdot \overline{w_{q, i}}+\overline{w_{p q, i}^{2}}-\overline{w_{p, i}^{2}} \cdot \overline{w_{q, i}}-2 \overline{w_{p q, i}} \cdot \overline{w_{p, i}}\right) \cdot
\end{aligned}
$$

By exchanging in the last equation $p$ by $q$ one gets a similar expression for the $\left\langle W_{p} W_{q}^{2}\right\rangle$. In Eqs. (7,9) the following notations are used:

$$
\begin{aligned}
& W_{\alpha \beta \gamma}^{a b c}=\overline{w_{a, \alpha}} \cdot \overline{w_{b, \beta}} \cdot \overline{w_{c, \gamma}}, \quad W_{\beta \gamma}^{(a b) c}=\overline{w_{a b, \beta}} \cdot \overline{w_{c, \gamma}}-\overline{w_{a, \beta}} \cdot \overline{w_{b, \beta}} \cdot \overline{w_{c, \gamma}}, \\
& \overline{w_{i, j}^{n}}=\frac{1}{\left\langle N_{j}\right\rangle} \int w_{i}^{n}(x) \rho_{j}(x) d x, \quad \overline{w_{p q, i}^{n}}=\frac{1}{\left\langle N_{i}\right\rangle} \int w_{p}^{n}(x) w_{q}(x) \rho_{i}(x) d x \\
& \overline{w_{p q r, i}}=\frac{1}{\left\langle N_{i}\right\rangle} \int w_{p}(x) w_{q}(x) w_{r}(x) \rho_{i}(x) d x .
\end{aligned}
$$

As it is evident from Eqs. (77 90 the third moments of the $W$-quantities $\left\langle W_{p}^{3}\right\rangle,\left\langle W_{p}^{2} W_{q}\right\rangle$, $\left\langle W_{q}^{2} W_{p}\right\rangle$, and $\left\langle W_{p} W_{q} W_{r}\right\rangle$ are just linear combinations of the unknown third moments $\left\langle N_{p}^{3}\right\rangle$, $\left\langle N_{p}^{2} N_{q}\right\rangle,\left\langle N_{q}^{2} N_{p}\right\rangle$, and $\left\langle N_{p} N_{q} N_{r}\right\rangle$ of the multiplicity distributions. In Eqs. (7) 9 the first moments $\left\langle N_{j}\right\rangle$ and second moments $\left\langle N_{j}^{2}\right\rangle,\left\langle N_{p} N_{q}\right\rangle$ are assumed to have already been determined, as it is discussed in Section III. The coefficients 10 12 entering into the right-hand-side of Eqs. (7.9) are also calculable, since they are expressed in terms of experimentally measured $\rho_{j}(x)$-functions. The problem is then reduced to solving the system of linear equations for the third moments of the multiplicity distribution in terms of experimentally measured third moments of $W$-quantities. This approach gives a unique answer, because by construction the number of equations is equal to the number of unknown third moments. 
The method can be used to calculate any moments of the joint multiplicity distribution by deriving the corresponding set of linear equations.

\section{FIRST MOMENTS REEXAMINED}

As mentioned in sections III and III the first moments of multiplicity distributions can be directly obtained by integrating the corresponding $x$ distributions, $\rho_{j}(x)$. Here we consider the case when the shape of the $\rho_{j}(x)$ functions are known, whereas their normalizations are arbitrary. This may be important for the analysis of hadron production in a narrow acceptance region. One then uses the data from the wider kinematic region to enlarge statistics and thus to improve the quality of the $\rho_{j}(x)$ functions. We denote these arbitrary normalizations by $A_{j}$, i.e,

$$
\int d x \rho_{j}(x)=\left\langle A_{j}\right\rangle
$$

It should be emphasized that all identities defined in Eq. (2) should be recalculated using the distributions defined in Eq. (13).

Below we demonstrate that the identity method can also be applied to obtain the hadron multiplicities with these arbitrarily normalized mass distributions. The idea is to extract the unknown first moments of multiplicity distributions from first moments of $W$-quantities. Indeed, one can write:

$$
\begin{aligned}
& \left\langle W_{p}\right\rangle=\sum_{N_{1}=0}^{N_{1}=\infty} \sum_{N_{2}=0}^{N_{2}=\infty} \ldots \sum_{N_{k}=0}^{N_{k}=\infty} \mathcal{P}\left(N_{1}, N_{2}, \ldots, N_{k}\right) \int d x_{1}^{1} P_{1}\left(x_{1}^{1}\right) \ldots \int d x_{N_{1}}^{1} P_{1}\left(x_{N_{1}}^{1}\right) \\
& \times \int d x_{1}^{2} P_{2}\left(x_{1}^{2}\right) \ldots \int d x_{N_{2}}^{2} P_{2}\left(x_{N_{2}}^{2}\right) \times \ldots \times \int d x_{1}^{k} P_{k}\left(x_{1}^{k}\right) \ldots \int d x_{N_{k}}^{k} P_{k}\left(x_{N_{k}}^{k}\right) \\
& \times\left[w_{p}\left(x_{1}^{1}\right)+\ldots+w_{p}\left(x_{N_{1}}^{1}\right)+w_{p}\left(x_{1}^{2}\right)+\ldots+w_{p}\left(x_{N_{2}}^{2}\right)+w_{p}\left(x_{1}^{k}\right)+\ldots+w_{p}\left(x_{N_{k}}^{k}\right)\right] \\
& =\sum_{i=1}^{k}\left\langle N_{i}\right\rangle \overline{w_{p, i}},
\end{aligned}
$$

where the mass probability distribution functions are now defined as $P_{i}(x) \equiv \rho_{i}(x) /\left\langle A_{i}\right\rangle$, and

$$
\overline{w_{p, i}}=\frac{1}{\left\langle A_{i}\right\rangle} \int w_{p}(x) \rho_{i}(x) d x
$$


The equation above can be written in a matrix form:

$$
\left(\begin{array}{c}
\left\langle W_{1}\right\rangle \\
\left\langle W_{2}\right\rangle \\
\ldots \\
\left\langle W_{k}\right\rangle
\end{array}\right)=\left(\begin{array}{cccc}
\overline{w_{1,1}} & \overline{w_{1,2}} & \ldots & \overline{w_{1, k}} \\
\overline{w_{2,1}} & \overline{w_{2,2}} & \ldots & \overline{w_{2, k}} \\
\ldots & \ldots & \ldots & \ldots \\
\overline{w_{k, 1}} & \overline{w_{k, 2}} & \ldots & \overline{w_{k, k}}
\end{array}\right)\left(\begin{array}{c}
\left\langle N_{1}\right\rangle \\
\left\langle N_{2}\right\rangle \\
\ldots \\
\left\langle N_{k}\right\rangle
\end{array}\right)
$$

The only unknowns in Eq. (16) are the first moments $\left\langle N_{j}\right\rangle$ which can easily be obtained by matrix inversion.

Note that when $\left\langle A_{i}\right\rangle=\left\langle N_{i}\right\rangle$ the right-hand-side of Eq. 14 yields:

$$
\sum_{i=1}^{k}\left\langle N_{i}\right\rangle \overline{w_{p, i}}=\sum_{i=1}^{k} \int \frac{\rho_{p} \cdot \rho_{i}}{\rho} d x=\int \rho_{p} d x=\left\langle N_{p}\right\rangle .
$$

Therefore, in this special case the first moments of the multiplicity distributions are identical to the first moments of $W$-quantities, $\left\langle W_{p}\right\rangle=\left\langle N_{p}\right\rangle$, similar to the case of CI, cf. Eq. (6).

\section{TEST ON SIMULATED DATA}

In this section we apply formulae (7-9) to simulated data. As already mentioned in section II we assume that particle identification is achieved by measuring a quantity $x$. In each event we generate three different particle species ${ }^{2}$ called proton $(\mathrm{p})$, kaon $(\mathrm{K})$, and pion $(\pi)$. Furthermore, the multiplicity distributions of these particles are simulated according to the Poisson distribution with different mean values. Consequently, the input information to our simulation is an inclusive (i.e., summed over all events and all tracks) distribution of the PID variable $x$ for each particle (see shaded histograms in upper panel of Figure 1) and mean multiplicities of particles. In our example these multiplicities are 8, 6 and 4 for protons, pions and kaons respectively. The simulation process comprises the following: (i) in each event we randomly generate from the Poisson distribution particle multiplicities using their mean values; (ii) we generate the quantity $x$ for each particle $j$ in an event from the inclusive distribution $\rho_{j}(x)$ of $x$ quantity for the corresponding particle. Using this simulated data we reconstruct all second and third moments of the multiplicity distributions. For each measurement $x$ in an event we

\footnotetext{
${ }^{2}$ Note that the method does not have any limitation on the number of particle species.
} 

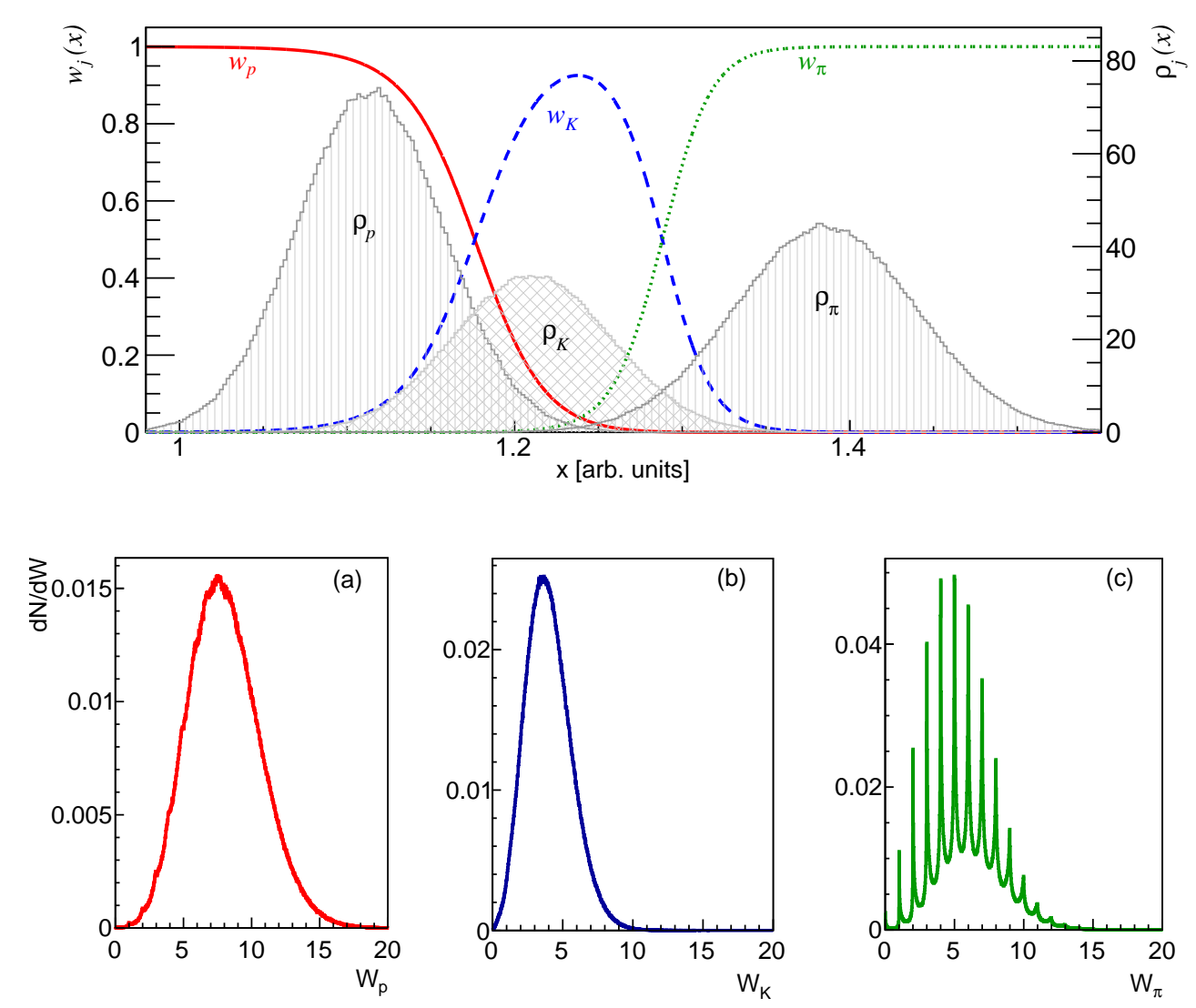

FIG. 1. (Color online) Upper panel: The inclusive distribution $\rho_{j}(x)$ of the particle identification variable $x$ for protons, kaons and pions, used as an input to the simulation, are depicted with shaded histograms. The distributions of identities $w_{j}(x)$ for protons, kaons and pions are presented with red solid, blue dashed and green dotted lines respectively. Lower panel: The distribution of event variables $W_{j}$ with $j$ standing for (a) protons, (b) kaons and (c) pions.

calculate identity variables $w_{j}(x)$ according to Eq. (2), where $j$ stands for the particle type. Next, for each particle we determine the event variable $W_{j}$ (see Eq. (4)). Figure 1 (upper panel) shows the distributions of the identity variables $w_{j}$ for different particle species. In the lower panel of Fig. 1 distributions of the $W_{j}$ quantities are plotted. As seen from Fig. 1 the $W_{\pi}$ distribution exhibits evident structures. This is not surprising as by construction the latter is nothing else but the original multiplicity distribution smeared because of non-ideal particle identification, i.e., because of the overlapping $\rho_{j}(x)$ distributions of different particles. These 
structures are better seen for $W_{\pi}$ because the $x$ distribution of the pions is well separated from those of protons and kaons as illustrated by the shaded histograms in the upper panel of Fig. 1 .

Finally using Eqs. (7-9) we reconstruct all third moments of the particles from the corresponding moments of the $\mathrm{W}$ quantities. We would like to emphasize that all second moments needed for reconstruction of the third moments are obtained using the formulae of Ref. [10]. The results are summarized in Table I, where the Poisson values are calculated using the moment generating function of the Poisson distribution, $M(t)=e^{-<N>} e^{<N>e^{t}}$ :

$$
\begin{gathered}
<N^{2}>=M^{\prime \prime}(t=0)=<N>^{2}+<N> \\
<N^{3}>=M^{\prime \prime \prime}(t=0)=<N>^{3}+3<N^{2}>+<N>.
\end{gathered}
$$

In case of vanishing correlations between particles (as assumed in our simulation) one finds:

$$
\begin{gathered}
<N_{1}^{2} N_{2}>=\left(<N_{1}>^{2}+<N_{1}>\right)<N_{2}> \\
<N_{1} N_{2}>=<N_{1}><N_{2}> \\
<N_{1} N_{2} N_{3}>=<N_{1}><N_{2}><N_{3}>
\end{gathered}
$$

The statistical errors of the reconstructed moments of the multiplicity distributions result from the errors on the parameters of the fitted distributions $\rho_{j}(x)$ of the identification observable $x$ and the errors of the $W_{j}$ quantities ${ }^{3}$. Typically these two sources of errors are correlated. Moreover, one can experimentally study fluctuation signals built up from several moments of the multiplicity distributions. The standard error propagation is complicated and inconvenient. We therefore follow the Monte Carlo error calculation approach. We first randomly subdivide the simulated data into $\mathrm{n}$ subsamples and for each subsample reconstruct the moments $M_{n}$ listed in Table I. In the second step we calculate the statistical error of each moment $M$ as:

$$
\sigma_{<M>}=\frac{\sigma}{\sqrt{n}}
$$

${ }^{3}$ Contributions to the uncertainty of the reconstructed moments due to the assumptions on the functional representation of $\rho_{j}(x)$ depend on the features of the experiment and their discussion is beyond the scope of this paper. 


\begin{tabular}{|c|c|c|c|}
\hline \hline Moments & Reconstructed with the identity method & Generated values & Poisson values \\
\hline$\left\langle p^{2}\right\rangle$ & $72.0429 \pm 0.0122$ & 72.0452 & 72 \\
\hline$\left\langle\pi^{2}\right\rangle$ & $42.0033 \pm 0.0073$ & 42.0011 & 42 \\
\hline$\left\langle K^{2}\right\rangle$ & $20.0066 \pm 0.0052$ & 20.0057 & 20 \\
\hline$\langle p \pi\rangle$ & $48.0178 \pm 0.007$ & 48.0184 & 48 \\
\hline$\langle p K\rangle$ & $32.0147 \pm 0.0057$ & 32.0154 & 32 \\
\hline$\langle\pi K\rangle$ & $24.0026 \pm 0.0033$ & 24.002 & 24 \\
\hline \hline$\left\langle p^{3}\right\rangle$ & $712.6391 \pm 0.1925$ & 712.6718 & 712 \\
\hline$\left\langle\pi^{3}\right\rangle$ & $330.0547 \pm 0.0878$ & 330.0316 & 330 \\
\hline$\left\langle K^{3}\right\rangle$ & $116.045 \pm 0.0501$ & 116.0372 & 116 \\
\hline$\left\langle p^{2} \pi\right\rangle$ & $432.307 \pm 0.0954$ & 432.3185 & 432 \\
\hline$\left\langle p^{2} K\right\rangle$ & $288.1968 \pm 0.0710$ & 288.2081 & 288 \\
\hline$\left\langle\pi^{2} K\right\rangle$ & $168.0083 \pm 0.033$ & 167.9971 & 168 \\
\hline$\left\langle\pi^{2} p\right\rangle$ & $336.155 \pm 0.0786$ & 336.1525 & 336 \\
\hline$\left\langle K^{2} p\right\rangle$ & $160.0986 \pm 0.0518$ & 160.0875 & 160 \\
\hline$\left\langle K^{2} \pi\right\rangle$ & $120.0275 \pm 0.0320$ & & 120 \\
\hline$\langle p \pi K\rangle$ & $192.0765 \pm 0.0379$ & & \\
\hline \hline
\end{tabular}

TABLE I. Results from the identity method for the $15 \cdot 10^{6}$ simulated events with mean multiplicities corresponding to $\langle p\rangle=8,<\pi>=6$ and $\langle K\rangle=4$. All second moments (upper part) listed in the table are reconstructed according to Ref. [10], all third moments (lower part) according to the formulae of Section IV. The statistical errors were obtained from the Monte Carlo method using Eqs.(23-25). Moments directly calculated from the simulated multiplicity distributions are shown in the third column and the Poisson values obtained from Eqs.(18-22) are listed in the last column. 


\begin{tabular}{|c|c|c|c|}
\hline \hline Moments & Reconstructed with the identity method & Generated values & Poisson values \\
\hline$\left\langle p^{2}\right\rangle$ & $72.0216 \pm 0.0172$ & 72.0198 & 72 \\
\hline$\left\langle\pi^{2}\right\rangle$ & $42.0108 \pm 0.0066$ & 42.0054 & 42 \\
\hline$\left\langle K^{2}\right\rangle$ & $72.0177 \pm 0.0144$ & 72.0198 & 72 \\
\hline$\langle p \pi\rangle$ & $48.0154 \pm 0.0082$ & 48.0096 & 48 \\
\hline$\langle p K\rangle$ & $72.0166 \pm 0.0143$ & 72.0198 & 72 \\
\hline$\langle\pi K\rangle$ & $48.0074 \pm 0.0072$ & 48.0096 & 48 \\
\hline \hline$\left\langle p^{3}\right\rangle$ & $712.3745 \pm 0.2572$ & 712.3739 & 712 \\
\hline$\left\langle\pi^{3}\right\rangle$ & $330.0893 \pm 0.0806$ & 330.0229 & 330 \\
\hline$\left\langle K^{3}\right\rangle$ & $712.3043 \pm 0.2167$ & 712.3739 & 712 \\
\hline$\left\langle p^{2} \pi\right\rangle$ & $432.2096 \pm 0.1246$ & 432.1387 & 432 \\
\hline$\left\langle p^{2} K\right\rangle$ & $712.3465 \pm 0.2249$ & 712.3739 & 712 \\
\hline$\left\langle\pi^{2} K\right\rangle$ & $336.0526 \pm 0.0744$ & 336.0604 & 336 \\
\hline$\left\langle\pi^{2} p\right\rangle$ & $336.1102 \pm 0.0838$ & 432.1386739 & 336 \\
\hline$\left\langle K^{2} p\right\rangle$ & $712.3191 \pm 0.2079$ & & 412 \\
\hline$\left\langle K^{2} \pi\right\rangle$ & $432.0899 \pm 0.1054$ & & 432 \\
\hline$\langle p \pi K\rangle$ & $432.1456 \pm 0.1079$ & & 4387 \\
\hline \hline
\end{tabular}

TABLE II. Results from the identity method for the $15 \cdot 10^{6}$ simulated events with mean multiplicities corresponding to $\langle p>=8,<\pi>=6$. In each event the number of kaons is taken to be equal to the number of protons in order to probe the method in presence of correlations. All second moments (upper part) listed in the table are reconstructed according to Ref. [10], all third moments (lower part) according to the formulae of Section IV. The statistical errors were obtained from the Monte Carlo method using Eqs.(23-25). Moments directly calculated from the simulated multiplicity distributions are shown in the third column and the Poisson values are listed in the last column. Note that for protons and kaons Eqs.(20-22) are not satisfied anymore due to the introduced correlations. 
where

$$
<M>=\frac{1}{n} \sum M_{n},
$$

and

$$
\sigma=\sqrt{\frac{\sum\left(M_{i}-<M>\right)^{2}}{n-1}} .
$$

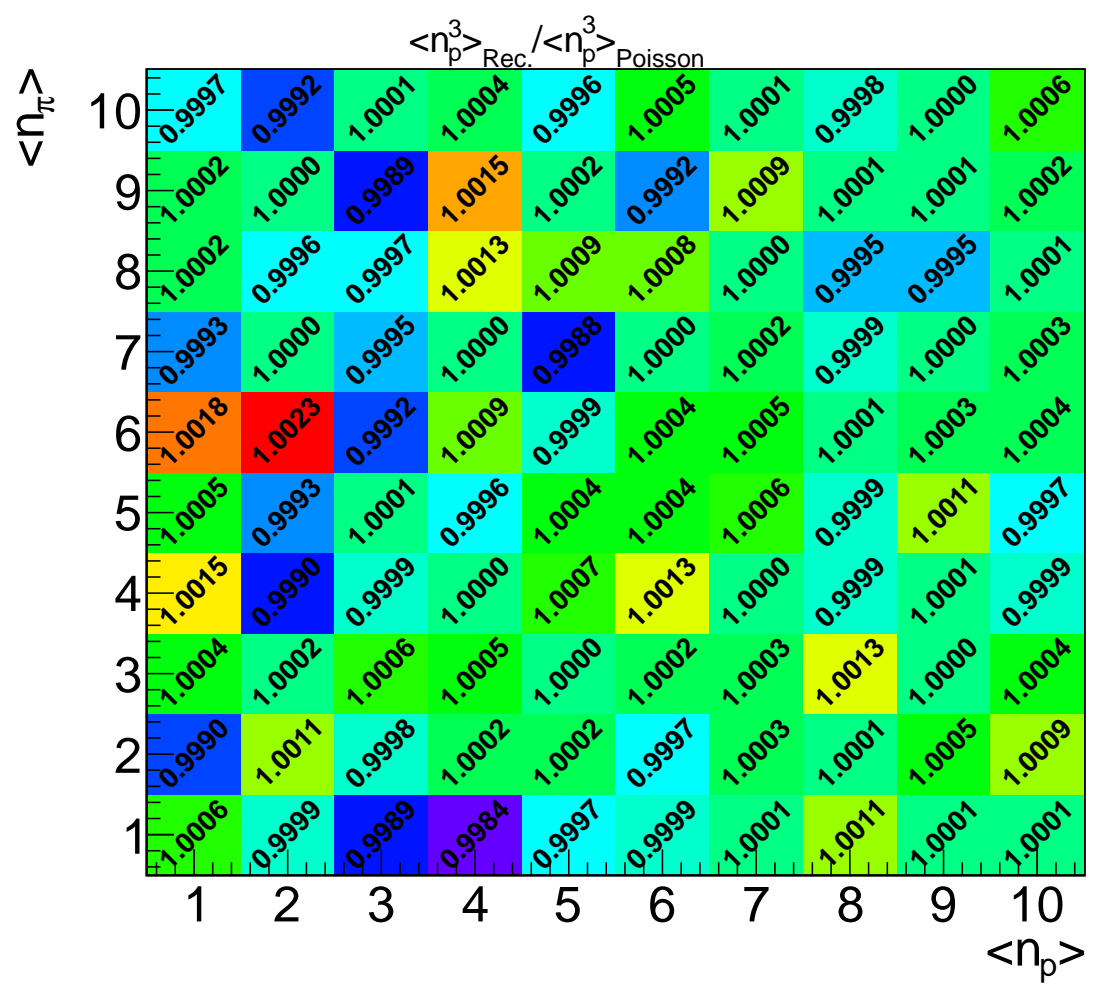

FIG. 2. (Color online) Result of 100 simulations, each with $6 \cdot 10^{6}$ events, with different mean multiplicities of protons and pions while the multiplicity of kaons in each event is taken to be equal to the number of protons. In each box the ratio of the reconstructed third moment $\left\langle n_{p}^{3}\right\rangle$ to the Poisson expectation (see Eq. (19p) is presented.

The reconstructed moments, using the identity method, presented in Table 1 are in agreement with the generated moments within statistical uncertainties. Next, in order to demonstrate that the method works also in case of strong correlations, we introduced an artificial correlation 
between protons and kaons. In each event we simulate the number of the protons and pions from the corresponding Poisson distributions as described above. However, the number of the kaons in each event is taken to be equal to the number of protons. The reconstructed moments from this simulation for $15 \cdot 10^{6}$ events with the mean multiplicities corresponding to 8 and 6 for the protons and pions respectively are presented in Table II. Note that the mean multiplicity of kaons is the same as for protons as described above. As evident from the Table II the reconstructed moments are again in reasonable agreement with the generated moments. However, for protons and kaons Eqs.(20-22) are not satisfied anymore due to the introduced correlations.

In Fig. 2 we demonstrate the result of 100 simulations, with $6 \cdot 10^{6}$ events in each, with different mean multiplicities of protons and pions. The number of the kaons in each event is taken to be equal to the number of protons, in order to induce a correlation between particles (see the text above). In each box the ratio of the reconstructed third moment $<n_{p}^{3}>$ of the proton multiplicity distribution to the Poisson expectation calculated using Eq. (19) is presented. One finds a high precision of the identity method independent of the values of average multiplicities.

\section{SUMMARY}

In summary, we extended the identity method proposed in Refs. [9, 10] to third moments of unknown multiplicity distributions. We introduce the event quantities $W_{j}$ depending on the single-particle identity variables $w_{j}$ according to Eq. (4). Any moments of these quantities $\left\langle W_{p} \ldots W_{q}\right\rangle$ can be measured experimentally. The problem of finding the second moments $\left\langle N_{j}^{2}\right\rangle$ and $\left\langle N_{p} N_{q}\right\rangle$ was solved in Ref. [10]. The third moments of $W$-quantities are found to be linear combinations of the unknown third moments $\left\langle N_{p}^{3}\right\rangle,\left\langle N_{p}^{2} N_{q}\right\rangle,\left\langle N_{q}^{2} N_{p}\right\rangle$ and $\left\langle N_{p} N_{q} N_{r}\right\rangle$. The

first moments $\left\langle N_{j}\right\rangle$ and second moments $\left\langle N_{j}^{2}\right\rangle,\left\langle N_{p} N_{q}\right\rangle$ entering into these relations are already known. We are confident that the procedure can be used for obtaining any higher moments of the multiplicity distributions by deriving the corresponding set of linear equations. We thus construct an iterative procedure in which all moments of the multiplicity distribution of the $n^{\text {th }}$ order are calculated from measured $n^{\text {th }}$ order moments of the $W$-quantities. By doing so, we get 
a unique solution, as the number of linear equations in each step is always equal to the number of the unknown moments. We presented an explicit test of the method using simulated events including the case of strong correlations between particles. Note that the higher moments (e.g., third and fourth moments of pion and proton multiplicity distributions [11, 12]) can reveal the effects of the QCD critical point with higher sensitivity in the event-by-event multiplicity fluctuations in nucleus-nucleus collisions

\section{ACKNOWLEDGMENTS}

We would like to thank Marek Gaździcki, Peter Seyboth and Herbert Ströbele for fruitful discussions and comments. The work of M. I. G. was supported by Humboldt Foundation and by the Program of Fundamental Research of the Department of Physics and Astronomy of NAS, Ukraine. A. R. gratefully acknowledges the support by the German Research Foundation (DFG grant GA 1480/2.1).

[1] V. Koch, in Relativistic Heavy Ion Physics, Landold-Börnstein Volume I/23, edited by R. Stock (Springer, Berlin, 2010).

[2] M. Stephanov, K. Rajagopal, and E. Shuryak, Phys. Rev. Lett. 81, 4816 (1998); Phys. Rev. D 60, 114028 (1999); M. Stephanov, Acta Phys. Polon. B 35, 2939 (2004).

[3] V. Koch, A. Majumder and J. Randrup, Phys. Rev. Lett. 95, 182301 (2005).

[4] V. Koch, A. Majumder and J. Randrup, Phys. Rev. C 72, 064903 (2005).

[5] S. V. Afanasev et al. [NA49 Collaboration], Phys. Rev. Lett. 86, 1965 (2001).

[6] C. Alt et al. [NA49 Collaboration], Phys. Rev. C 79, 044910 (2009).

[7] D. Kresan [for CBM Collaboration and NA49 Collaboration], PoS CPOD2009, 031 (2009).

[8] B. I. Abelev et al. [STAR Collaboration], Phys. Rev. Lett. 103, 092301 (2009).

[9] M. Gaździcki, K. Grebieszkow, M. Maćkowiak, and S. Mrówczyński, Phys. Rev. C 83, 054907 (2011).

[10] M.I. Gorenstein, Phys. Rev. C 84, 024902 (2011). 
[11] M.A. Stephanov, Phys. Rev. Lett. 107, 052301 (2011); Phys. Rev. Lett. 102, 032301 (2009).

[12] C. Athanasion, K. Rajagopal, and M. Stephanov, Phys. Rev. D 82, 074008 (2010). 